\title{
REVISIONES
}

\section{Intoxicación por plomo en humanos}

\author{
Lead effects on humans
}

\author{
Pedro A Poma ${ }^{1}$ \\ ${ }^{1}$ Profesor en Medicina Clínica. University of Illinois. Chicago, EE UU.
}

\begin{abstract}
Resumen
El plomo es un metal que se ha usado extensamente desde la antigüedad, por lo que se le puede demostrar en la mayoría de personas. Este metal afecta sistemas, órganos y tejidos y su efecto puede ser proporcional a la cantidad presente en el organismo. Pero los umbrales de sus efectos tóxicos varían en diferentes individuos. Los niños generalmente absorben una mayor proporción del plomo y con un efecto más severo que los adultos, porque están en un proceso activo de desarrollo y por ciertas características fisiológicas, patológicas y de conducta. Los servicios de salud pública pueden identificar áreas en que la población tiene un riesgo mayor de intoxicación con plomo y establecer condiciones para el despistaje, identificación temprana y tratamiento de las personas afectadas. La determinación de plomo en sangre venosa es la prueba más sensible de exposición al plomo. Se recomienda que los niveles en sangre se mantengan debajo de $10 \mu \mathrm{g} / \mathrm{dL}$. Este artículo revisa las fuentes de contaminación, efectos, diagnóstico y tratamiento del plomo.
\end{abstract}

Palabras clave: Intoxicación por plomo; prevención, toxicidad, diagnóstico.

\begin{abstract}
Lead, a metal that has been extensively used since antiquity, can be found in the majority of persons. This metal affects systems, organs, and tissues. The thresholds for its toxic effects vary from individual to individual. Probably because they are in the process of development and due to certain physiological, pathological y behavioral characteristics children usually absorb a greater proportion of lead with more severe outcomes than adults. Public health services can identify areas where people has a higher risk for lead intoxication and establish requirements for screening, early diagnosis and adequate treatment. The determination of lead in venous blood is the most sensitive test for the evaluation of lead exposure. Experts recommend maintaining a level below $10 \mu \mathrm{g} / \mathrm{dL}$. This article reviews origin, effects, diagnosis and treatment of lead intoxication.
\end{abstract}

Key words: Lead poisoning; prevention, toxicity, diagnosis.
El plomo es un metal blando, gris azulado, estable y resistente a la corrosión. Sin embargo, cuando forma parte de tuberías, conexiones o soldaduras, en presencia de agua ligeramente ácida puede contaminar el agua potable ${ }^{(1)}$. El plomo no conduce bien la electricidad, posee una temperatura de fusión baja, por lo que se le utiliza (fusible) en algunas partes de las instalaciones eléctricas; cuando la carga eléctrica excede un nivel predeterminado, se funde e interrumpe la transmisión eléctrica. Este metal también ofrece protección contra la radiación, por lo que se emplea en mandiles de trabajadores de salud y en las paredes de los salones de diagnóstico y tratamiento radiológico. Desde la antigüedad, el plomo ha sido usado extensamente y se le puede encontrar en casi todas las personas, aún a niveles elevados ${ }^{(2)}$

Este metal existe en forma inorgánica y orgánica. La forma inorgánica puede encontrarse en las pinturas, tierra, polvo y en otros productos de manufactura. Los gases de la combustión de la gasolina, a la que se le ha agregado plomo, contiene la forma orgánica del metal (plomo tetra etilo); nuestro cuerpo absorbe esta forma más fácilmente, por lo que resulta más tóxica que la forma inorgánica ${ }^{(1)}$. Con la eliminación del plomo de la gasolina en la mayoría de países la contaminación con la forma orgánica de plomo se limita a casos de contaminación ocupacional.

El Perú es uno de los cinco mayores productores de plomo en el mundo, con un volumen de exportación que continúa aumentando (Figura 1). Las minas de las que se extrae plomo, las fundiciones y sus alrededores naturalmente sufren una mayor contaminación. A los servicios de salud pública y a los profesionales les corresponde un papel activo en la educación de políticos, empresarios y del público en general, para así disminuir la posibilidad de efectos adversos asociados con la presencia, uso y comercio de este metal. Este artículo revisa las fuentes de contaminación, los efectos del plomo en el organismo humano, la evaluación diagnóstica y el tratamiento de sus efectos tóxicos.

Fuentes de contaminación

En EE UU, la contaminación con plomo está asociada con la presencia de pintura que contiene plomo en construcciones vie- jas, con lugares de trabajo que usan plomo (fábrica de baterías), con agua potable, comidas y bebidas contaminadas, con juguetes, medicinas tradicionales ${ }^{(3)}$, cosméticos y con la tierra, polvo, agua, aire de las cercanías de minas y fundiciones ${ }^{(1)}$ (Tabla 1 ).

En EEUU, se eliminó casi completamente el plomo de las pinturas en 1977 (0,06\%) (1). Pero, aunque la pintura esté cubierta con otras capas de pintura sin plomo, este metal puede entrar al ambiente del hogar cuando la pintura se descascara, debido a fricción o impacto o durante reparaciones actuales o pasadas ${ }^{(4)}$. Los trabajadores de construcción o puentes se exponen a las capas de pinturas que estas estructuras han recibido a través de los años, antes de las nuevas regulaciones. Los trabajadores antes de pintar un puente o un edificio o casa tienden a remover las capas de pinturas del metal (puente) o de paredes o techos que están descascarados — los resanan — antes de aplicar las nuevas capas de pintura. Este procedimiento remueve las capas de pinturas nuevas, sin plomo, exponiendo las capas de pinturas antiguas con plomo a la piel, vías respiratorias, ropa y herramientas de los trabajadores. 


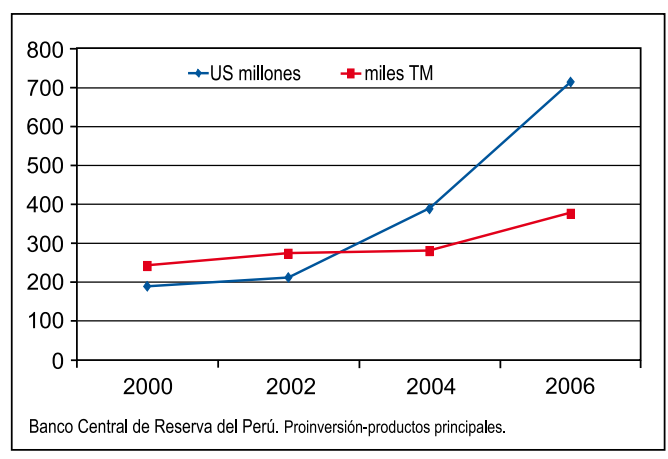

Figura 1. Exportación de plomo, 2000-2006.

Tabla 1. Fuentes de contaminación de plomo.

- Minas y fundiciones.
- Soldaduras y tuberías.
- Contenedores y latas.
- Pinturas.
- Gasolina.
- Lugares de trabajo con productos con plomo.
- Contaminación de agua, comidas, bebidas.
- Polvo y tierra en las viviendas y alrededores.
- Alimentos, comidas, remedios, cosméticos, joyas,
juguetes importados.

Además, el plomo de esas estructuras contamina la tierra alrededor de la casa o los trabajadores llevan el plomo en sus ropas a sus casas (bañándose, cambiándose de ropa y zapatos; antes de regresar a casa, pueden prevenir esta exposición secundaria), aunque algunos de estos negocios que utilizan el plomo funcionan en la casa, donde la posibilidad de contaminación es mayor (Tabla 2).

El plomo también puede contaminar productos que normalmente no contienen este mineral, como las comidas, agua o licor. Esta contaminación puede ocurrir durante la producción, embalaje o almacenamiento de los productos. Por ejemplo, las raíces del vegetal pueden extraer el mineral del suelo o el mineral en la atmósfera puede depositarse en las hojas de los vegetales, o los equipos de cortar o moler pueden estar contaminados ${ }^{(5)}$. Las pinturas que se utiliza en las latas o cerámica o cristales en que se envasa el producto pueden contener

Tabla 2. Plomo en el lugar de trabajo

\begin{tabular}{l} 
- Minas, refinerías y fundiciones. \\
- Reparación de automóviles. \\
- Fábrica de baterías. \\
- Trabajo en reparación de puentes. \\
- Construcción civil, especialmente renovación. \\
- Lugares de práctica de tiro, municiones. \\
- Gasolineras. \\
- Producción de vidrio, plásticos, caucho, otros productos \\
- Pontaminados. \\
- Imprentas. \\
- Policias. \\
- Construcción de barcos. \\
- Soldadores y cortadores de acero. \\
\hline
\end{tabular}

plomo ${ }^{(5)}$. El plomo se usa en baterías o radiadores de automóviles, pintura de puentes, computadoras, productos de jebe o plástico, joyería o cerámica, cristales y pewter (mezcla de estaño y plomo). Los remedios tradicionales mejicanos utilizados para tratar el empacho, que contienen plomo, se les conoce con el nombre de azarcón, greta ${ }^{(3)}$, Alarcón, coral, liga, María Luisa y rueda. En las comunidades asiáticas, se conoce los productos que contienen plomo con los nombres de ba-baw-san, bai goli, chuifong, ghasard, kandu, tokuwan ${ }^{(1)}$.

\section{Absorción del plomo}

La absorción del plomo depende del estado de salud, nutrición y edad de la persona. Los adultos generalmente absorben 20\% del plomo que ingieren y casi todo ese plomo es inhalado. La mayor parte del plomo que ingresa al cuerpo es excretado por la orina o a través de la bilis por las heces. La forma más común de plomo, la inorgánica, no es metabolizada en el hígado; mientras tanto, el plomo orgánico ingerido (presente en aditivos de la gasolina) se absorbe casi en su totalidad y es metabolizado en el hígado. El plomo en la gasolina puede ingresar a través de la piel. La extracción del plomo del subsuelo (minas) y las emisiones de las fundiciones afectan tanto a niños como a adultos ${ }^{(6,7)}$. La ingestión de polvo contaminado o de alimentos, agua o alcohol contaminados es la forma más común de ingreso del plomo al organismo. Los niños absorben una proporción mayor que los adultos. La inhalación es la vía de ingreso más común en personas que utilizan este metal en sus ocupaciones ${ }^{(1)}$.

El plomo que no es excretado permanece en el cuerpo por periodos prolongados y se intercambia entre 3 compartimientos -sangre, huesos y dientes- que contienen casi la totalidad del plomo, y en otros tejidos, como el hígado, riñones, pulmones, cerebro, bazo, músculos y corazón ${ }^{(1)}$. El plomo almacenado en los huesos y dientes puede volver a entrar a la circulación durante periodos de deficiencia de calcio, como el embarazo, lactancia y osteoporosis ${ }^{(1)}$.

Comparado con los adultos, los niños absorben más el plomo que ingieren (Figura 2). Los niños a menudo se colocan las manos y los objetos en la boca, ingieren más tierra o polvo contaminado. Además, muchos niños tienden a comer productos no alimenticios (pica), su frecuencia respiratoria es más alta, respiran más volumen por kilos de peso y, como son más pequeños, están más cerca del aire contaminado con el polvo, así como con emisiones del subsuelo. Mientras menos edad tienen, el intestino absorbe más plomo, 5 a 10 veces, que niños mayores y los adultos, especialmente con el estómago vacío ${ }^{(8)}$. La absorción intestinal de plomo en niños aumenta en casos de deficiencia de hierro, calcio y zinc, que son condiciones comunes ${ }^{(1)}$. Todas estas condiciones favorecen un mayor riesgo de toxicidad en los niños.

En EE UU, desde 1991, se recomienda que niños con un nivel de diez microgramos por decilitro $(10 \mu \mathrm{g} / \mathrm{dL})$ de plomo en sangre requieren acción inmediata (CDC, Center for Disease Control and Prevention, Atlanta, GA). Se indica que todos los niños que viven en zonas reconocidas como de riesgo alto de intoxicación tengan pruebas de sangre y que sus niveles se mantengan bajo $10 \mu \mathrm{g} / \mathrm{dL}$, porque varios estudios han demostrado alteraciones neurológicas y de conducta, aún a niveles más bajos que

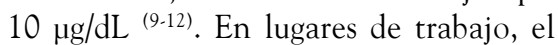
límite permisible promedio de plomo es 50 $\mu \mathrm{g} / \mathrm{m} 3$ por la jornada de 8 horas (OSHA, Occupational Safety Health Administration, Department of Labor, Washington, DC). Pero, OSHA requiere pruebas de plomo en sangre para trabajadores expuestos a concentraciones de $\geq 30 \mu \mathrm{g} / \mathrm{dL}$ en el aire por más de 30 días al año. Si el nivel sanguíneo es $>40 \mu \mathrm{g} / \mathrm{dL}$, la compañía debe notificar al empleado por escrito ofreciéndole una evaluación médica. Si el nivel sanguíneo llega a $60 \mu \mathrm{g} / \mathrm{dL}$ o resulta en un promedio de $\geq 50 \mu \mathrm{g} / \mathrm{dL}$ en $\geq 3$ pruebas, la compañía debe relevarlo de esa exposición excesiva, manteniendo su sueldo y tiempo de servicio hasta un nivel $<40 \mu \mathrm{g} / \mathrm{dL}$.

Se considera no contaminada a la tierra o polvo con concentraciones de plomo $<50$

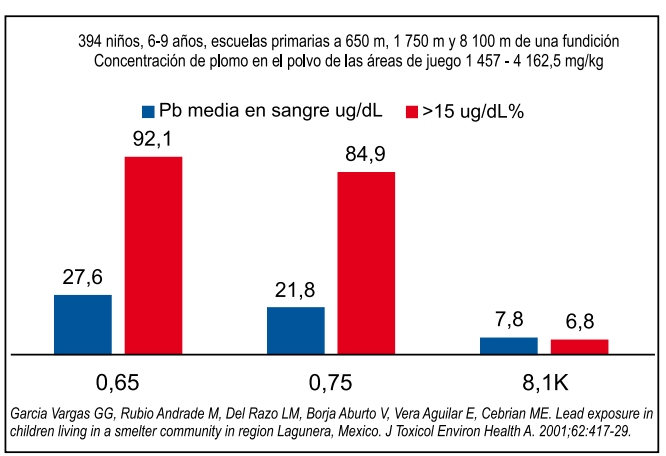

Figura 2. Pb en sangre en niños - distancia de la fundición. 
ppm (partes por millón); pero, en muchas áreas urbanas las concentraciones de plomo exceden 200 ppm ${ }^{(13)}$. La EPA (Environmental Protection Agency, Washington, DC) recomienda 400 ppm de peso en áreas accesibles al juego de niños y a 1200 ppm en áreas no accesibles al juego. La EPA también recomienda ausencia completa del plomo en el agua potable y $15 \mu \mathrm{g} / \mathrm{dL}$ como nivel que requiere acción. La FDA (Food and Drug Administration, Department of Health and Human Services, Washington, DC) recomienda acción si se encuentra el plomo a un nivel 0,5 $\mu \mathrm{g} / \mathrm{dL}$ en alimentos destinados para infantes y niños y ha eliminado el uso de envases que usan soldadura de plomo.

La sangre solo lleva una pequeña cantidad del plomo total, pero sirve como medio de transporte para la distribución de este metal en el cuerpo y su excreción. Se estima que el tiempo medio de vida del plomo en la sangre es de 28 a 36 días ${ }^{(14)}$. Casi todo el plomo en sangre (99\%) se encuentra en los eritrocitos, el resto en el plasma ${ }^{(15,16)}$.

En adultos, los huesos y dientes contienen $94 \%$ y en niños $73 \%$ de la cantidad total de plomo del cuerpo ${ }^{(17)}$. El plomo no se distribuye uniformemente en estos tejidos, pero tiende a acumularse en regiones con la calcificación más activa durante la exposición. En los niños, el plomo tiende a acumularse en el hueso trabecular, mientras que en los adultos se acumula en ambas cortical y trabecular ${ }^{(18)}$. Parece que existen 2 compartimientos fisiológicos en los huesos: el compartimiento inerte (almacena plomo por décadas) y el compartimiento lábil (intercambia fácilmente su contenido de plomo con la sangre) ${ }^{(1)}$. Bajo ciertas circunstancias, el plomo del compartimiento inerte vuelve a entrar a la sangre y a otros tejidos. La movilización del plomo del compartimiento inerte a la sangre aumenta en condiciones de deficiencia de calcio, como durante el embarazo, lactancia, menopausia, condiciones de estrés, enfermedades crónicas, hipertiroidismo, enfermedad renal, fracturas y edad avanzada. Este componente inerte permanece como una fuente endógena de plomo que puede causar niveles elevados de plomo en sangre años después que la exposición al metal ha pasado. Por lo que, los síntomas pueden aparecer en ausencia de exposición actual al plomo. En la mayoría de los casos, los niveles de plomo en sangre representan una mezcla de exposición actual y contribución endógena de exposiciones anteriores. Pero, una exposición aguda puede acompañarse brevemente de niveles sanguíneos muy elevados y causar síntomas de toxicidad.

\section{Efectos del plomo}

El plomo afecta todos los órganos y sistemas. Actúa como agonista o antagonista de las acciones del calcio y se relaciona con proteínas que poseen los grupos sulfidrílicos, amina, fosfato y carboxilo ${ }^{(1)}$. El nivel sanguíneo de plomo materno aumenta el riesgo fetal y de alteraciones neurológicas en los recién nacidos. Los embarazos con niveles elevados de plomo en la sangre tienen un riesgo mayor de partos prematuros, abortos espontáneos, muertes fetales y de recién nacidos con peso bajo para su edad gestacional $^{(1)}$.

En niños, se ha asociado la exposición al plomo con ausencias más frecuentes a la escuela, menor rendimiento escolar, intervalos de reacción prolongados y coordinación mano-ocular disminuida (13). La inmadurez fisiológica de fetos e infantes (hasta la edad de 36 meses) aumenta el riesgo de que el plomo penetre al sistema nervioso central, lo que puede resultar en alteraciones neurológicas o de conducta permanentes. El plomo también puede afectar los sistemas renal, endocrino y sanguíneo. No existe un nivel de plomo en sangre que se pueda considerar inocuo en niños. La ausencia de síntomas no excluye el envenenamiento por plomo. Algunos estudios sugieren que el plomo continúa ejerciendo efectos negativos en la conducta social juvenil ${ }^{(19,20)}$. Los efectos inmediatos del plomo son neurológicos, pero el envenenamiento en la infancia puede conducir más tarde a problemas renales, hipertensión arterial y problemas de la reproducción ${ }^{(1)}$.

\section{Efectos neurológicos}

Los problemas neurológicos se pueden presentar aún en individuos con niveles de plomo en sangre considerados seguros $(<10$ $\mu \mathrm{g} / \mathrm{dL})^{(9-13)}$. Individuos aún sin síntomas, especialmente niños, pueden tener daño neurológico ${ }^{(1)}$. En los niños, la exposición aguda a dosis altas de plomo puede causar encefalopatía, con la presencia de ataxia, convulsiones, hiperirritabilidad, estupor, coma y muerte. Varios estudios en niños se refieren a diferentes niveles sanguíneos de plomo asociados con encefalopatía, pero un nivel $\geq 70 \mu \mathrm{g} / \mathrm{dL}$ indica un riesgo alto de presentar la complicación. Este nivel está asociado con daño neurológico o alteraciones de conducta a largo plazo, aunque el niño aún no presente síntomas y signos de encefalopatía ${ }^{(1)}$.
Algunos estudios muestran que, por cada $10 \mu \mathrm{g} / \mathrm{dL}$ de aumento de plomo en sangre, el cociente de Inteligencia baja 4 a 7 puntos ${ }^{(21-23)}$. La exposición al plomo también está asociada con otros problemas neurosicológicos, disminución de la atención con hiperactividad, sordera, alteraciones del balance y de los nervios periféricos. Algunos de estos defectos persisten en la edad adulta $^{(1)}$.

Los individuos expuestos al plomo como adultos pueden presentar las mismas complicaciones que presentan los niños, pero a niveles más altos de plomo en sangre. Por ejemplo, la encefalopatía puede ocurrir con $460 \mu \mathrm{g} / \mathrm{dL}^{(24)}$.

Los síntomas precursores de la encefalopatía incluyen somnolencia, irritabilidad, disminución de la atención y memoria y presencia de tremores, que pueden ocurrir con niveles más bajos de plomo en sangre (Tabla 3). En la realidad clínica, los síntomas y signos no necesariamente ocurren en la forma esperada; algunos síntomas ocurren a niveles más bajos, otros a niveles más altos. Existen otros síntomas que

Tabla 3. Signos y síntomas de una exposición continua a plomo.

\section{Exposición muy baja}

Disminución de memoria, aprendizaje, cociente de inteligencia, habilidad verbal, atención, pronunciación y audición. Signos de hiperactividad.

Exposición leve

Parestesias, mialgias, fatiga leve, irritabilidad, letargia, molestias abdominales.

Exposición moderada

Artralgias, fatiga general, dificultad de concentración, cansancio muscular, tremor, cefaleas, dolor abdominal difuso, vómitos, pérdida de peso, estreñimiento.

\section{Exposición alta}

Parestesias, parálisis, encefalopatía (puede causar convulsiones, alteración de la conciencia, coma y muerte), línea azul oscura en los encías, cólicos intermitentes y severos.

pueden ocurrir a niveles de 40 a $120 \mu \mathrm{g} / \mathrm{dL}$; estos incluyen disminución de libido, de dexteridad manual, habilidad de entender, intervalo de reacción, actividad visual motora, cociente de inteligencia, memoria y concentración. Esta persona también puede presentar depresión, cefaleas, cambios del estado de ánimo, dolores de cabeza, mareos, fatiga, impotencia, irritabilidad, letargia, nerviosidad, malestar general, parestesias y debilidad. Además, se describe problemas de equilibrio postural y alteraciones de los nervios periféricos. Los trabajadores expuestos a niveles altos de plomo muestran parálisis del nervio radial (Figura 3), debido a disminución de la conducción del nervio y debilidad muscular ${ }^{(1)}$. 


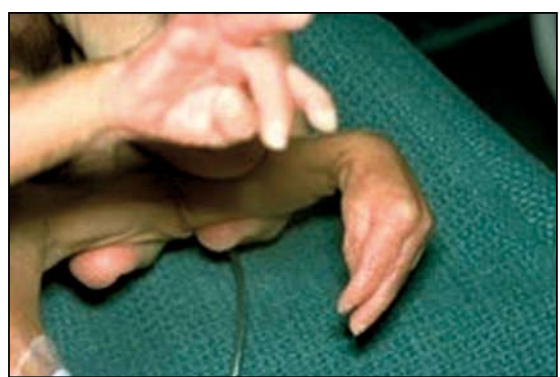

Figura 3. Parálisis del nervio radial izquierdo.

\section{Efectos renales}

La severidad de la exposición al plomo se relaciona directamente con los efectos en el riñón. Una exposición severa por un periodo breve se la asocia con alteraciones reversibles de la función tubular proximal — glicosuria, aminoaciduria, hiperfosfaturia (1). Sin embargo, exposiciones continuas o repetidas pueden conducir a nefropatía crónica (nefritis intersticial), que es generalmente irreversible (Figura 4). No se conoce

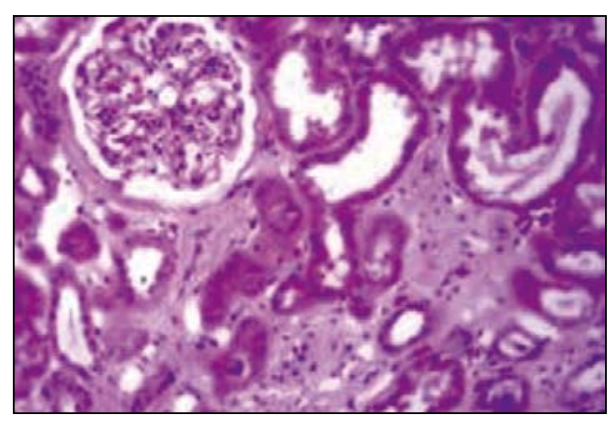

Figura 4. Biopsia renal muestra atrofia tubular no especifica y fibrosis intersticial. Note la ausencia de infiltrado intersticial; un glomérulo es normal.

el nivel mínimo de plomo que causa esta complicación, pero varias comunicaciones indican $>60 \mu \mathrm{g} / \mathrm{dL}$ (1). Tampoco existe alguna prueba de diagnóstico de daño renal temprano. Pero, para evaluar la condición renal se emplea la creatinina en sangre y la prueba de la depuración de la creatinina. Algunos estudios encuentran un aumento acelerado de la creatinina o disminución de la depuración de la creatinina cuando los niveles de plomo en la sangre son $<60$ $\mu \mathrm{g} / \mathrm{dL}^{(25,26)}$. Los efectos del plomo en ocasiones anteriores, por ejemplo durante la infancia, pueden favorecer la disminución de la función renal o la progresión a la fase crónica. En niños, los efectos renales de la intoxicación aguda parecen reversibles y la recuperación ocurre generalmente dentro de los dos meses de tratamiento (27). El tratamiento parece que previene la progresión de la nefropatía aguda a nefritis intersticial crónica ${ }^{(28)}$. La enfermedad renal puede mantenerse asintomática hasta sus estadios tardíos, a menos que se la descubra mediante pruebas de laboratorio.

La exposición al plomo también puede causar hipertensión arterial de origen renal. Con la disminución de la función renal, la excreción de ácido úrico disminuye, lo que determina hiperuricemia y síntomas de gota. Un estudio determinó que más de la mitad de los pacientes que sufría de nefropatía relacionada con plomo presentaba síntomas de gota ${ }^{(29)}$. Los pacientes con este tipo de gota presentan ataques menos frecuentes que los que sufren de la gota clásica. Este tipo de gota puede ocurrir en mujeres de edad reproductiva, lo que es raro en la gota clásica ${ }^{(30)}$. Los casos de gota por intoxicación por plomo afectan al riñón más frecuentemente y la complicación es más severa que en los casos de gota clásica (31).

\section{Anemia}

La presencia del plomo disminuye la producción del núcleo heme, lo que afecta la habilidad del organismo de producir hemoglobina. El plomo inhibe la dehidratasa del ácido d-amino levulínico y la actividad de la ferrochelatasa. La ferrochelatasa cataliza la inserción del hierro a la protoporfirina IX y es muy sensible al efecto del plomo. Con la disminución de la actividad de esta enzima aumenta la protoporfirina del glóbulo rojo. En presencia del plomo, aumenta el ácido damino levulínico, en sangre y plasma, así como la protoporfirina libre ${ }^{(1)}$. Se estima que un nivel de plomo en sangre de 50 $\mu \mathrm{g} / \mathrm{dL}$ en adultos con exposición ocupacional y de alrededor de $40 \mu \mathrm{g} / \mathrm{dL}$ en niños, representan el umbral de anemia, aunque otros estudios en niños sugieren un umbral más bajo $(25 \mu \mathrm{g} / \mathrm{dL})^{(1)}$. La determinación de protoporfirina en eritrocitos no es una prueba suficientemente sensitiva como para usarla en el despistaje de intoxicación por plomo, especialmente en niños ${ }^{(1)}$.

El plomo puede causar dos tipos de anemia, a menudo acompañadas con inclusiones basófilas de los eritrocitos jóvenes (Figura 5). Una exposición severa aguda se asocia con anemia hemolítica. La anemia no es una manifestación temprana de la intoxicación con plomo; solo se hace evidente con exposiciones altas y prolongadas ${ }^{(1)}$. En casos de exposición crónica, el plomo produce anemia, porque interfiere con la síntesis del núcleo heme y disminuye el promedio de vida de los eritrocitos.
La anemia es hipocrómica y normocítica o microcítica asociada a reticulocitosis. La alteración de la síntesis del núcleo heme también afecta otros procesos biológicos del sistema nervioso, renal, endocrino y hepático ${ }^{(1)}$.

En niños existe una correlación inversa entre los niveles de plomo en la sangre y los niveles de vitamina D. El plomo previene la conversión de la vitamina $\mathrm{D}$ a 1, 24. dihidroxivitamina D. Esta hormona, que mantiene el equilibrio del calcio intracelular y extracelular, además puede limitar el crecimiento, maduración y desarrollo de los huesos y dientes. Estos efectos son evidentes en casos de exposición crónica con niveles sanguíneos elevados $(62 \mu \mathrm{g} / \mathrm{dL})$ y desnutrición crónica, especialmente relacionada a deficiencia de calcio, fosforo y vitamina $\mathrm{D}$ (32).

En casos severos de envenenamiento, los niños o adultos pueden quejarse de cólicos severos, que pueden ser considerados equivocadamente como apendicitis o abdomen agudo.

\section{Efectos cardiovasculares}

La hipertensión arterial está relaciona con varios factores de riesgo; estos factores incluyen la edad, peso corporal, dieta y actividad física. La exposición al plomo puede ser otro factor que contribuye al desarrollo de la hipertensión. Aunque la exposición a niveles bajos o moderados de plomo (nivel sanguíneo $<30 \mu \mathrm{g} / \mathrm{dL}$ ) solo muestra una relación mínima, los niveles más elevados, generalmente relacionados a contactos ocupacionales, aumentan el riesgo de hipertensión arterial y enfermedad cerebrovascular. Comparados con los controles, los adultos que sufrieron de envenenamiento con plomo durante la infancia tienen un riesgo mayor de hipertensión ${ }^{(33)}$. Esta asociación también ha sido encontrada en estudios de población con niveles de plomo en sangre $<30 \mu \mathrm{g} / \mathrm{dL}^{(34)}$. Se estima que la intoxicación con plomo causa hasta el 2\% de casos de hipertensión arterial ${ }^{(1)}$.

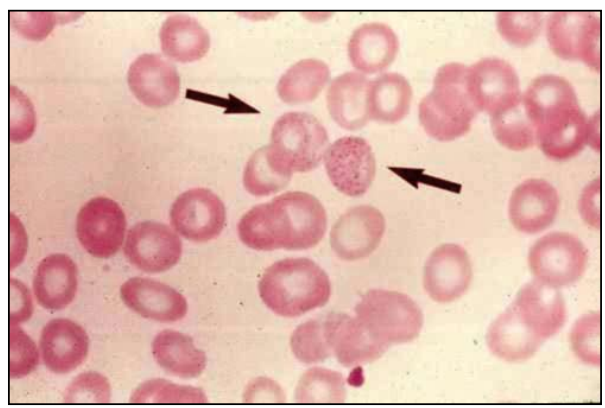

Figura 5. Eritrocitos con inclusiones basófilas. 


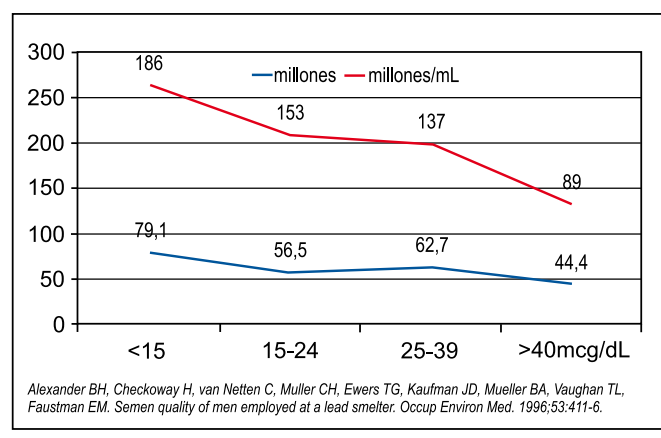

Figura 6. Calidad del semen, de acuerdo al nivel del plomo.

\section{Sistema reproductor}

La exposición al plomo causa disminución de la cuenta total y aumento en la proporción de espermatozoides anormales (35-38) (Figura 6). Los efectos comienzan con niveles de alrededor de $40 \mu \mathrm{g} / \mathrm{dL}$. La exposición crónica, aparte del efecto de una exposición aguda, también disminuye la concentración, cuenta total y motilidad de los espermatozoides ${ }^{(35)}$. Se desconoce la duración de estos efectos nocivos, después que cesa la exposición al plomo.

No se conoce con certeza si el plomo a niveles bajos afecta el resultado de los embarazos. Pero, existe una asociación clara entre la exposición a nivel ocupacional y las consecuencias en el embarazo. Algunos estudios, que comparan a mujeres embarazadas con niveles sanguíneos más elevados de plomo que viven cerca de fundiciones con otras que viven lejos, muestran aumento de la frecuencia de abortos espontáneos (39) (Figura 7), muertes fetales ${ }^{(40)}$ y partos prematuros ${ }^{(41,42)}$. Pero no todos los estudios encuentran esta asociación ${ }^{(43)}$.

Durante el embarazo, el plomo entra libremente al compartimiento fetal, por lo que afecta la viabilidad fetal y el desarrollo del niño durante la vida extrauterina. Aún con niveles sanguíneos bajos $(14 \mu \mathrm{g} / \mathrm{dL})$, la exposición al plomo puede aumentar el riesgo de partos prematuros y de recién nacidos con peso menor que el esperado. Un estudio encontró una asociación del

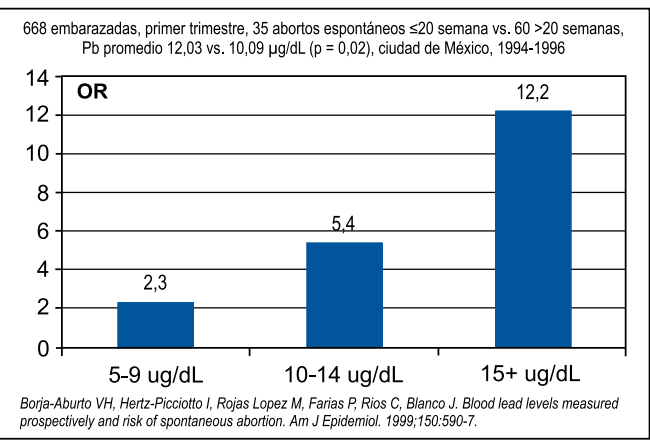

Figura 7. Abortos y niveles de $\mathrm{Pb}$ en sangre. plomo con anomalías congénitas, como testículos no descendidos y complicaciones de la piel ${ }^{(44)}$. Pero, no se ha demostrado anomalías congénitas mayores relacionadas con el plomo ${ }^{(40)}$.

La proporción de niños con problemas de aprendizaje es mayor en los hijos de padres que fueron expuestos al plomo durante su infancia ${ }^{(45)}$.

\section{Evaluación diagnóstica}

Los clínicos pueden identificar a los individuos expuestos a niveles peligrosos de plomo, antes que se produzcan síntomas de intoxicación severa. El reconocimiento de un individuo puede facilitar la identificación de muchos otros que sufren de la misma exposición. El Departamento de Salud de la cuidad de Chicago recomienda pruebas de sangre para plomo cada 6 meses en todos los niños. Estas pruebas las comienzan entre los 6 y 9 meses de edad, con la identificación del tipo de riesgo de envenenamiento que el niño presenta. Se debe averiguar el origen del agua que toman, el tipo de tubería que tienen, la condición de los animales caseros (también son afectados), posible exposición familiar (tipo de trabajo), uso de remedios caseros, la posibilidad de que la casa tenga pinturas con plomo, remodelación de las viviendas, ocupación familiar en el domicilio, uso de cerámica e historia de familiares intoxicados.

La intoxicación es más seria en niños menores de 6 años. Se debe evaluar a todo niño con problemas del desarrollo, de conducta, dificultades de pronunciación o que pueden haber sido expuestos, y también a sus hermanos, compañeros de casa y de juego. Se puede instruir a la comunidad de estos riesgos y a los padres durante el embarazo.

El examen físico debe incluir evaluación de cambios de conducta y de los sistemas neurológico, hematológico, cardiovascular (presión arterial), gastrointestinal y renal.

Se debe evaluar en los niños el progreso de la audición, pronunciación y momentos claves del desarrollo. Como la deficiencia de calcio y hierro facilitan la absorción del plomo y agravan la pica, es muy importante evaluar el estado nutricional. Existe una continuación de signos y síntomas relacionados con los niveles y la duración de la exposición (Tabla 3). La susceptibilidad de los individuos varía, por lo que el inicio de los síntomas también puede variar. La disminución de habilidades puede ocurrir con niveles entre 10 y $15 \mu \mathrm{g} / \mathrm{dL}$; los síntomas de intoxicación ocurren entre los 35 y $50 \mu \mathrm{g} / \mathrm{dL}$ en niños y 40-60 $\mu \mathrm{g} / \mathrm{dL}$ en adultos; y, la toxicidad severa, entre los $\geq 70 \mu \mathrm{g} / \mathrm{dL}$ en niños $\mathrm{y} \geq 100$ $\mu \mathrm{g} / \mathrm{dL}$ en adultos.

Algunas de las alteraciones sanguíneas pueden también presentarse en otras enfermedades. En casos de anemia microcítica, si el nivel del plomo en sangre es $<25 \mu \mathrm{g} / \mathrm{dL}$, la anemia se debe a deficiencia de hierro o a una hemoglobinopatía. Existen dos enfermedades raras (porfiria aguda intermitente y coproporfiria), que también resultan de alteraciones del núcleo heme (como la intoxicación con plomo). Algunas personas con intoxicación con plomo solo son tratadas por neuropatía periférica o por el síndrome carpal, lo que pospone un tratamiento adecuado. Cuando se demora el diagnóstico de intoxicación con plomo, los síntomas abdominales pueden ser considerados como abdomen agudo y llevar al paciente al quirófano.

\section{Laboratorio}

La determinación de plomo en sangre venosa es la prueba que representa mejor la severidad de la exposición reciente y crónica a este metal. Pero, en individuos con exposición muy severa o crónica, el nivel en la prueba en sangre venosa no representa la cantidad total de plomo en su organismo. La mayor parte del plomo se almacena en los huesos, por lo que el individuo puede presentar niveles normales en la sangre. Los individuos con niveles elevados de plomo en sangre requieren un hemograma completo, hemoglobina, hematocrito y determinación de hierro y ferritina.

Cuando un individuo ha tragado joyas, peso de cortinas, residuos de pintura que contienen plomo, las radiografías de abdomen pueden mostrar la presencia de cuerpos extraños en el tracto digestivo. En niños, las radiografías de huesos largos pueden mostrar las líneas de plomo - líneas de mayor densidad en las metáfisis de crecimiento (Figura 8). Ésta no es una prueba que se utiliza para el diagnóstico, pero su presencia indica exposición crónica.

\section{Tratamiento}

En general, en casos de intoxicación por plomo, lo más importante es remover la fuente de la intoxicación. En los EE UU, los departamentos locales de salud pública están involucrados con estos procesos. Sus 


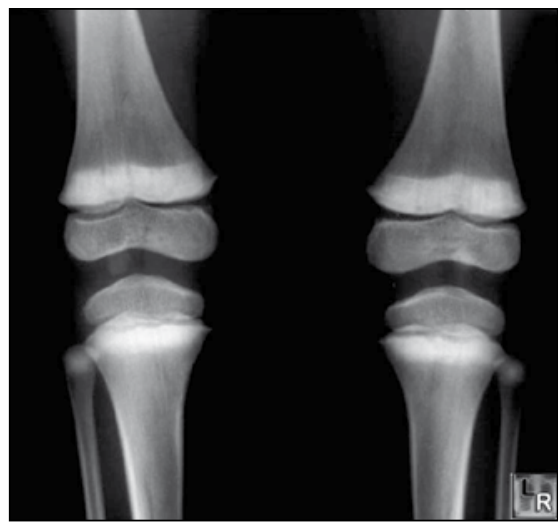

Figura 8. Radiografía de las rodillas. Note las líneas de plomo-densidad aumentada en las metáfisis de crecimiento.

trabajadores de salud tienen la experiencia para identificar productos caseros que pueden ser la fuente de la contaminación, proveen la educación familiar, ofrecen centros de referencia, tratamiento y seguimiento.

\section{Quelación}

En individuos con niveles de plomo sanguíneos elevados, los agentes quelantes se unen al plomo en la sangre, facilitando su eliminación por la bilis y orina, lo que reduce la cantidad total de plomo en el cuerpo y sus efectos tóxicos (Tabla 4). No se recomienda estos agentes para personas con niveles de plomo $<45 \mu \mathrm{g} / \mathrm{dL}$. En las personas con exposición alta al plomo y cuyos niveles sanguíneos son bajos, tampoco se usa estos agentes, porque el riesgo de movilizar el plomo es más severo que los beneficios del tratamiento. En estos casos, se recomienda una dieta rica en calcio, para evitar que su deficiencia movilice el plomo de los huesos.

Para este tratamiento, se recomienda edetato disodio cálcico (versenato de calcio disódico, $\mathrm{CaNa}_{2}$ EDTA). Otros productos incluyen al dimercaprol (British antilewisite, 2,3-dimercaptro-propanol), d-penicilamina (cuprimina, 3-mercapto-D-valina) y Succimer (Chemet, ácido meso-2,3-dimercaptosuccínico). En niños, se debe evitar el ácido etileno diamino tetraacético disódico

Tabla 4. Guía para el tratamiento, de acuerdo al nivel sanguíneo de plomo.

\begin{tabular}{cl}
\hline $\begin{array}{c}\text { Niveles sanguíneos } \\
\text { de plomo }(\mu \mathrm{g} / \mathrm{dL})\end{array}$ & \multicolumn{1}{c}{ Tratamiento } \\
\hline 10 a 19 & $\begin{array}{l}\text { Educación, investigar la fuente, consultas, } \\
\text { repita la prueba en } 3 \text { meses, resultados 15 a 19, } \\
\text { considere guía de } 20 \text { a } 44 .\end{array}$ \\
20 a 44 & $\begin{array}{l}\text { Educación, evaluación clínica y de laboratorio, } \\
\text { intervención agresiva de la fuente, repita pruebas } \\
\text { en 1 a 2 meses. } \\
45 \text { a } 69\end{array}$ \\
$\begin{array}{l}\text { Educación, coordinación y manejo en los dos dias } \\
\text { siguiente, tratamiento y repita las pruebas mensualmente. } \\
\text { Esta es una emergencia; evaluación y diagnóstico } \\
\text { inmediato, hospitalización para tratamiento. }\end{array}$ \\
\hline
\end{tabular}

( $\left.\mathrm{Na}_{2} \mathrm{EDTA}\right)$, porque puede causar hipocalcemia y paro cardiaco fatal ${ }^{\left({ }^{(4)}\right)}$. Los individuos que reciben este tratamiento no deben regresar a su domicilio ni los adultos a su trabajo, hasta que se descubra y se elimine la fuente de contaminación. Así también se identifica otros individuos afectados que requieren tratamiento y se evita un daño mayor en la comunidad. La solución no es fácil, sobre todo para personas pobres, quienes no tienen otro lugar donde vivir o dependen del trabajo para su subsistencia.

Como conclusión podemos decir que, en los últimos 20 años, en los EE UU, la proporción de niños con niveles de plomo en sangre de $\geq 10 \mu \mathrm{g} / \mathrm{dL}$ ha bajado más de $80 \%$, después de la eliminación del plomo de la gasolina, de la soldadura de plomo en latas que contienen alimentos y en las pinturas y otros productos de consumo. Ahora, son raras las muertes o los casos de niños con síntomas debidos a la intoxicación con plomo ${ }^{(47)}$. La dedicación de todos los sectores envueltos en la eliminación de este problema ha dado resultados.

\section{REFERENCIAS BIBLIOGRÁFICAS}

1. Agency of Toxic Substances and Disease Registry. Case studies in environmental medicine. Lead toxicity. US Department of Health and Human Services, Public Health Service. Atlanta, GA: The Agency; 2007.

2. Budd P, Montgomery J, Cox A, Krause P, Barreiro $B$, Thomas RG. The distribution of lead within ancient and modern human teeth: implications for long-term and historical exposure monitoring. Sci Total Environ. 1998;220:121-36.

3. Poma PA. A dangerous folk therapy. I Natl Med Assoc. 1984;76:387-9.

4. Lanphear BP, Matte TD, Rogers J, Cickner RP, Dietz $B$, Bornschein RL, et al. The contribution of lead-contaminated house dust and residential soil to children's blood lead levels. A pooled analysis of 12 epidemiologic studies. Environ Res. 1998;79:51-68.

5. Mushak P, Davis JM, Crocetti AF, Grant LD. Prenatal and postnatal effects of low-level lead exposure: integrated summary of a report to the U.S. Congress on childhood lead poisoning. Environ Res. 1989;50:11-36.

6. Kaul B, Sandhu RS, Depratt C, Reyes F. Follow-up screening of lead-poisoned children near an auto battery recycling plant, Haina, Dominican Republic. Environ Health Perspect. 1999;107:91720.

7. Lopez-Carrillo L, TorresSanchez L, Garrido F, PapaquiHernandez J, PalazuelosRendon E, Lopez-Cervantes M. Prevalence and determinants of lead intoxication in Mexican children of low socioeconomic status. Environ Health Perspect. 1996;104:1208-11.

8. James HM, Hilburn ME, Blair JA. Effects of meals and meal times on uptake of lead from the gastrointestinal tract in humans. Hum Toxicol. 1985;4:401-7.

9. Canfield RL, Henderson CR Jr, Cory-Slechta DA, Cox C, Jusko TA, Lanphear BP. Intellectual impairment in children with blood lead concentrations below 10 micrograms per deciliter. $\mathrm{N}$ Engl J Med. 2003;348:1517-26.

10.Lanphear BP, Hornung R, Khoury J, Yolton $K$, Baghurst P, Bellinger DC, et al. Low-level environmental lead exposure and children's intellectual function: an international pooled analysis. Environ Health Perspect. 2005;113:8949.

11. Lanphear BP, Dietrich K, Auinger P, Cox C. Cognitive deficits associated with blood lead concentrations $<10$ microg/dL in US children and adolescents. Public Health Rep. 2000;115:521-9.

12. Tellez-Rojo MM, Bellinger DC, Arroyo-Quiroz C, Lamadrid-Figueroa H, Mercado-Garcia A, Schnaas-Arrieta $L$, et al. Longitudinal associations between blood lead concentrations lower than 10 microg/dL and neurobehavioral development in environmentally exposed children in Mexico City. Pediatrics. 2006;118(2):e323-30.

13. American Academy of Pediatrics Committee on Environmental Health. Lead poisoning: from screening to primary prevention. Pediatrics. 1993;9 2:176-83.

14. Rabinowitz MB, Wetherill GW, Kopple JD. Kinetic analysis of lead metabolism in healthy humans. J Clin Invest. 1976;58:260-70.

15. de Silva PE. Determination of lead in plasma and studies on its relationship to lead in erythrocytes. Br J Ind Med. 1981;38:209-17.

16. Everson J, Patterson CC. "Ultra-clean" isotope dilution/mass spectrometic analyses for lead in human blood plasma indicated that most reported values are artificially high. Clin Chem. 1980;26:1603-7.

17. Barry PS. Concentrations of lead in the tissues of children. Br J Ind Med. 1981;38:61-71.

18. Aufderheide AC, Wittmers L Jr. Selected aspects of the spatial distribution of lead in bone. Neurotoxicology. 1992;13: 809-19.

19. Nevin R. How lead exposure relates to temporal changes in $\mathrm{QQ}$, violent crime, and unwed pregnancy. Environ Res. 2000;83:1-22.

20. Nevin R. Understanding international crime trends: the legacy of preschool lead exposure. Environ Res. 2007;104:315-36.

21. Schroeder SR, Hawk B, Otto DA, Mushak P, Hicks RE. Separating the effects of lead and social factors on IQ. Environ Res. 1985;38:144-54.

22. Lansdown R, Yule W, Urbanowicz MA, Hunter J. The relationship between blood-lead concentrations, intelligence, attainment and behaviour in a school population: the second London study. Int Arch Occup Environ Health. 1986;57:225-35. 
23. Hawk BA, Schroeder SR, Robinson G, Otto D, Mushak P, Keinbaum D, et al. Relation of lead and social factors to IQ of low-SES children: a partial replication. Am J Ment Defic. 1986;91:178-83.

24. Kehoe RA. The metabolism of lead in man in health and disease. Arch Environ Health. 1961; 2:41822.

25. Staessen JA, Lauwerys RR, Buchet JP, Bulpitt CJ, Rondia D, Vanrenterghem Y, Amery A. Impairment of renal function with increasing blood lead concentrations in the general population. The Cadmibel Study Group. N Engl J Med. 1992;327:151-6.

26. Tsaih SW, Korrick S, Schwartz J, Amarasiriwardena C, Aro A, Sparow D, et al. Lead, diabetes, hypertension, and renal function: the normative aging study. Environ Health Perspect. 2004;112:1178-82.

27. Chisolm JJ. Current status of lead exposure and poisoning in children. South Med J. 1976;69:52931.

28. Wedeen RP, D'Haese P, Van de Vyver FL, Verpooten GA, De Broe ME. Lead nephropathy. Am J Kidney Dis. 1986;8: 380-3.

29. Bennett WM. Lead nephropathy. Kidney Int. 1985;28:212-20.

30. Goyer RA. Mechanisms of lead and cadmium nephrotoxicity. Toxicol Lett. 1989;46:153-62.

31. Batuman V, Maesaka JK, Haddad B, Tepper E, Landy E, Wedeen RP. The role of lead in gout nephropathy. N Engl J Med. 1981;304:520-3.

32. Koo WW, Succop PA, Bornschein RL, Krug-Wispe SK, Steinchen JJ, Tsang RC, et al. Serum vitamin $D$ metabolites and bone mineralization in young children with chronic low to moderate lead exposure. Pediatrics. 1991;87:680-7.

33. Hu H. A 50-year follow-up of childhood plumbism. Hypertension, renal function, and hemoglobin levels among survivors. Am J Dis Child. 1991;145:6817.

34. Schwartz J. Lead, blood pressure, and cardiovascular disease in men. Arch Environ Health. 1995;50:31-7.

35. Alexander BH, Checkoway H, van Netten C, Muller CH, Ewers TG, Kaufman JD, et al. Semen quality of men employed at a lead smelter. Occup Environ Med. 1996;53:411-6.

36. Lerda D. Study of sperm characteristics in persons occupationally exposed to lead. Am J Ind Med. 1992;22:567-71.

37. Bonde JP, Joffe M, Apostoli P, Dale A, Kiss P, Spano $M$, et al. Sperm count and chromatin structure in men exposed to inorganic lead: lowest adverse effect levels. Occup Environ Med. 2002;59:23442.

38. Telisman S, Colak B, Pizent A, Jurasovic J, Cvitkovic $P$. Reproductive toxicity of low-level lead exposure in men. Environ Res. 2007;105:256-66.

39. Nordstrom S, Beckman L, Nordenson I. Occupational and environmental risks in and around a smelter in northern Sweden. V. Spontaneous abortion among female employees and decreased birth weight in their offspring. Hereditas. 1979;90:291-6.

40. McMichael AJ, Vimpani GV, Robertson EF, Baghurst PA, Clark PD. The Port Pirie cohort study: maternal blood lead and pregnancy outcome. J Epidemiol Community Health. 1986;40:18-25.

41. Torres-Sanchez LE, Berkowitz G, Lopez-Carrillo L, Torres-Arreola L, Rios C, Lopez-Cervantes M. Intrauterine lead exposure and preterm birth. Environ Res. 1999;81:297-301.

42. Jelliffe-Pawlowski LL, Miles SQ, Courtney JG, Materna B, Charlton V. Effect of magnitude and timing of maternal pregnancy blood lead $(\mathrm{Pb})$ levels on birth outcomes. J Perinatol.
2006;26:154-62.

43. Factor-Litvak P, Graziano JH, Kline JK, Popovac D, Mehmeti A, Ahmedi G, et al. A prospective study of birthweight and length of gestation in a population surrounding a lead smelter in Kosovo, Yugoslavia. Int J Epidemiol. 1991;20:722-8.

44. Needleman HL, Rabinowitz M, Leviton A, Linn S, Schoenbaum $S$. The relationship between prenatal exposure to lead and congenital anomalies. JAMA. 1984;251:2956-9.

45. Hu H. Knowledge of diagnosis and reproductive history among survivors of childhood plumbism. Am J Public Health. 1991;81:1070-2.

46. Centers for Disease Control and Prevention (CDC). Deaths associated with hypocalcemia from chelation therapy--Texas, Pennsylvania, and Oregon, 2003-2005. MMWR Morb Mortal Wkly Rep. 2006;55:204-7.

47. Lanphear BP, Dietrich KN, Berger O. Prevention of lead toxicity in US children. Ambul Pediatr. 2003;3:27-36.

Manuscrito recibido el 11 de febrero de 2008 y aceptado para publicación el 9 de abril de 2008.

Correspondencia:

Dr. Pedro A. Poma

University of Illinois at Chicago

505 N. Lake Shore Drive, Chicago,

Illinois 60611-6435 USA

Correo-e: palpoma@yahoo.com 